\title{
Electrophysiological characteristics of atrial tachycardia originating from the coronary sinus
}

\author{
Jie Wang ${ }^{1}$, Zhihong Zhao ${ }^{1 *}$, Xuebin Li $^{2}$, Jihong Guo ${ }^{2}$ \\ ${ }^{1}$ Department of Cardiology, Guang Anmen Hospital, China Academy of Chinese Medical Sciences, Beijing, China; \\ ${ }^{2}$ Department of Cardiology, People’s Hospital, University of Peking, Beijing, China. \\ Email: *zhihong zhao@126.com
}

Received 30 June 2011; revised 20 September 2011; accepted 18 November 2011.

\begin{abstract}
A 22-year-old girl was admitted to our cardiology institute with Permanent tachycardia in last 6 years for diagnostic assessment and therapy. Doppler echocardiography show structural of heart is normal, ECG revealed a varied resting rate from 120 to 140 bpm, 1:1 AV ratio and long $R P$ interval. $P$ wave morphology was negative on leads I, II, III, aVF, and V4 to V6, positive on lead V1, and diphasic 1 /2 on lead aVL. Electrophysiology (EP) study was performed, Although the exactly mapping was performed in left inferior pulmonary vein and mitral annulus, however, no target point was found ahead of CS1-2, the tried discharge was invalid. The ablation catheter was entered the coronary sinus to guide electrical isolation, curing the tachycardia. The ECG returned to normal sinus rhythm. Through three years follow up, no AT recurrence.
\end{abstract}

Keywords: Coronary Sinus; Atrial Tachycardia; Catheter Ablation

\section{INTRODUCTION}

Focal atrial tachycardia (at) is a relatively uncommon arrhythmia. Diagnosis of at is usually straightforward - simply by a routine ecg in most patients, only those with paroxysmal at may require holter monitoring. And this form of tachycardia can be successfully treated with radiofrequency catheter ablation (rfca) in long-term [1]. It has been demonstrated that specific anatomic structures form the preferential sites of origin of atrial tachycardia (at) [2-4]. In fact, when patients with failed ablation of focal at, an unusual location must be considered. There are few isolated reports of ats originating from the coronary sinus [5]. We present a case of 22-year old girl with focal atrial tachycardia, examined the electrophysiological and electrocardiography (ecg) features of this specific type of at arising from coronary sinus and the acute and long- term success rates of focal ablation.

\section{CASE REPORT}

Permanent tachycardia was diagnosed in a 22-year-old girl who was referred to our cardiology institute for diagnostic assessment and therapy.

Her heart rate was abnormally high in last 6 years with palpitation of exercise intolerance, her rhythm disorders was not previously identified by ecg. In our hospital, doppler echocardiography did not show a structural heart disease, ecg revealed a narrow complex tachycardia with a varied resting rate from 120 to $140 \mathrm{bpm}, 1: 1$ av ratio and long rp interval. P wave morphology was negative on leads i, ii, iii, avf, and v4 to v6, positive on lead v1, and diphasic $1 / 2$ on lead avl (Figure 1(a)).

Ep study was performed to identify the tachycardia mechanism and the earliest atrial depolarization during tachycardia recorded from the distal coronary sinus suggested focal left atrial tachycardia. Precision mapping was performed in left inferior pulmonary vein and mitral annulus, however, no target point was found ahead of cs1 - 2, the tried discharge was invalid. The ablation catheter was then entered the coronary sinus, which revealed a complex, fragmented, triphasic atrial electrogram with a very sharp first deflection preceding the beginning of the p wave by 6 msec ahead of cs1 - 2 (Figure 2(a)). $\mathrm{Rf}$ current application for 20 seconds in 50w at that level soon stopped the tachycardia after a transient slowing down over two cycles (Figure 2(b)).

After ablation, no atrial tachycardias were induced by isoprinosine i.v., and vena coronaria visualization found 20\% phlebostenosis at the place of discharge (Figures 3 (a)-(b). The ecg returned to normal sinus rhythm (Figure 1(b)).24-hour holter monitoring indicated a sinus rhythm. Through three years follow up, no at recurrence.

\section{DISCUSSION}

Electrophysiologists are often misled by varied patient's manifestations combined with confused ECG. All of which 


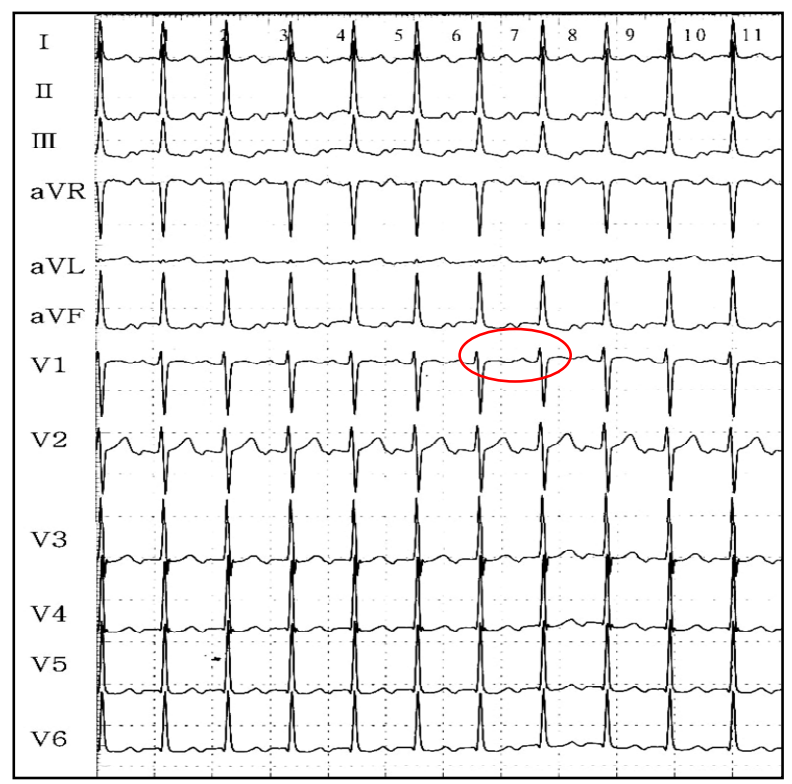

(a)

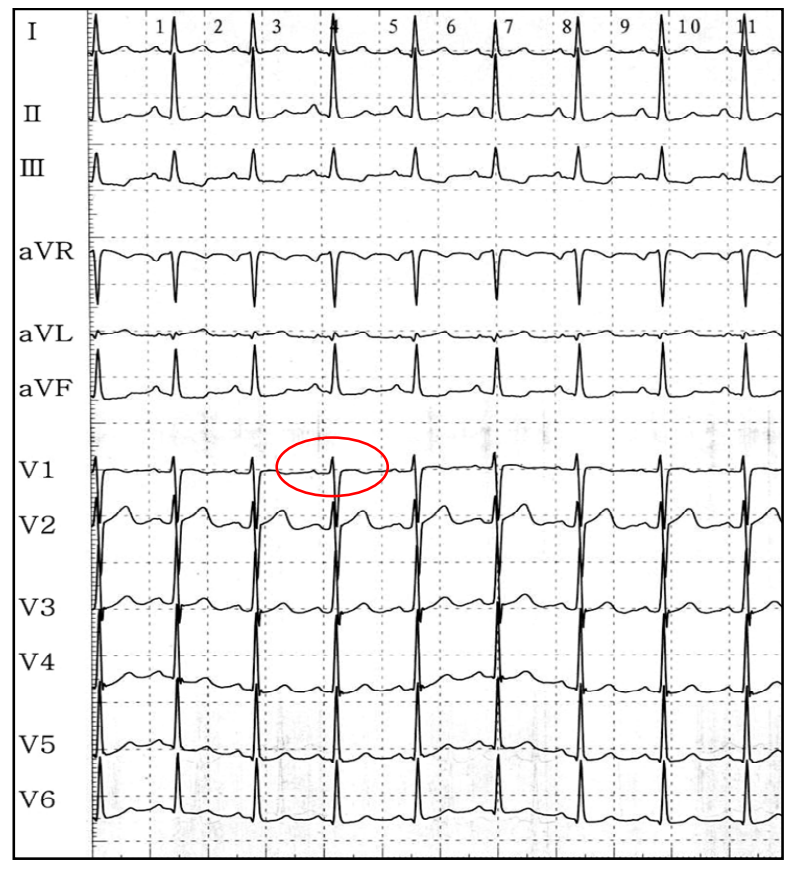

(b)

Figure 1. 12 lead ECG of the patients before (a), and after ablation; (b), the ECG returned to normal after ablation. Surface leads (I, II, V1) along with intracardiac electrogram recordings from the His-bundle, coronary sinus, and ablation catheters, the latter positioned at the successful ablation site just before energy delivery.

showed the complicated clinical manifestation of atrial tachycardia, made some patients suffered misdiagnosis and incorrect therapies by ostensible clinical situation. Precise diagnosis of those patients depended on the EP study, which is the most commonly used technique to locate the AT focus is endocardial activation mapping.

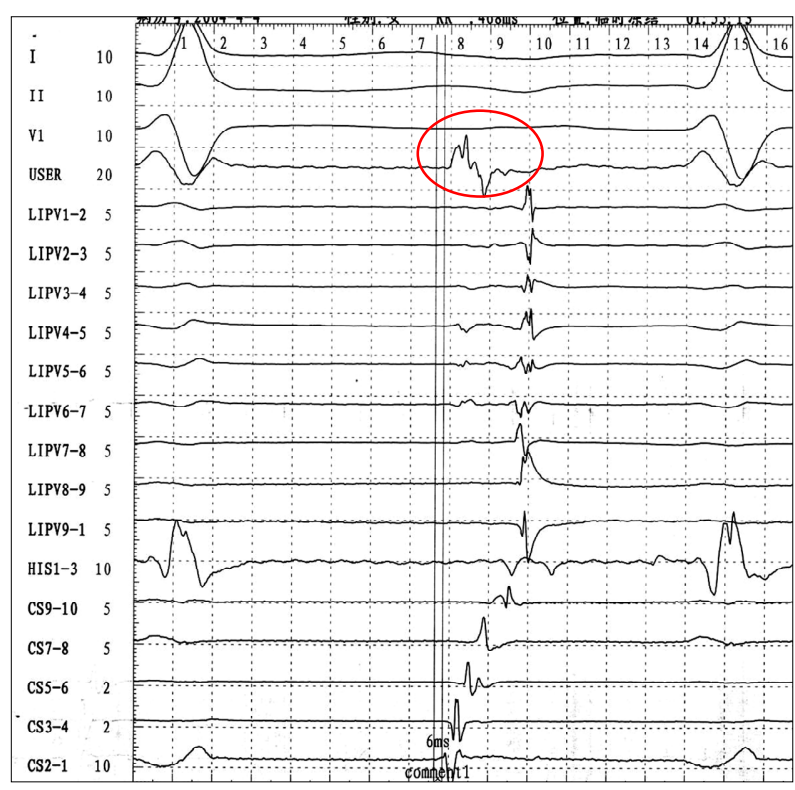

(a)

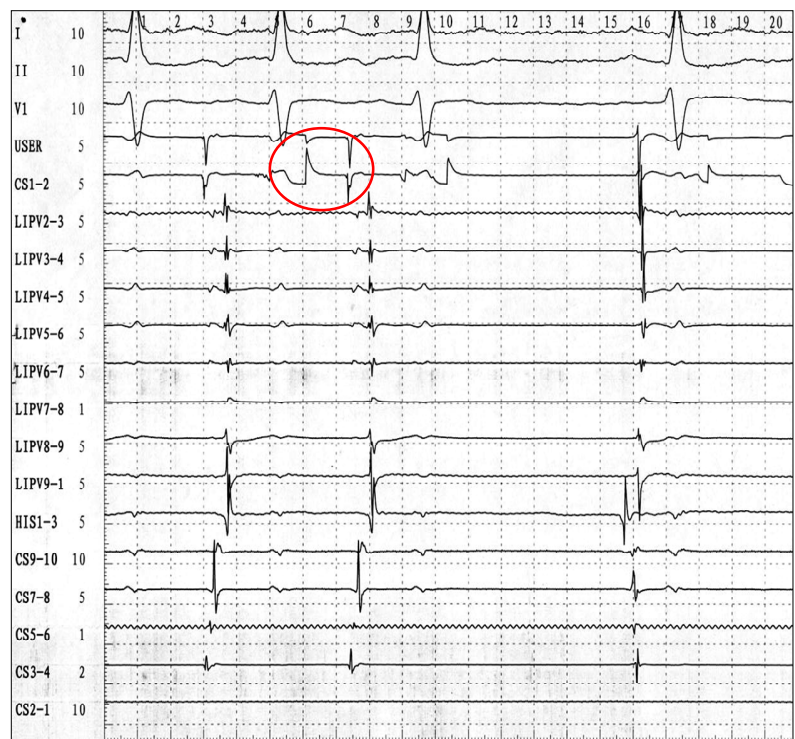

(b)

Figure 2. Fractionated electrogram preceding ectopic $\mathrm{P}$ wave from $6 \mathrm{msec}$ is clearly individualized at the distal bipole of the ablation catheter. Radiofrequency energy applied from within that site resulted in termination of the tachycardia within a few seconds ( $\mathrm{a}$ and $\mathrm{b}$ ).

With the advent of radiofrequency ablation this has become the major treatment of choice in patients with significant symptoms.

Focal AT is well-recognized that these foci do not occur randomly throughout the atria but tend to cluster at characteristic anatomic locations. In the right atrium they tend to cluster around the crista terminalis, the ostium of the coronary sinus, perinodal region, tricuspid annulus, right atrial well and right atrial appendage. In the left 


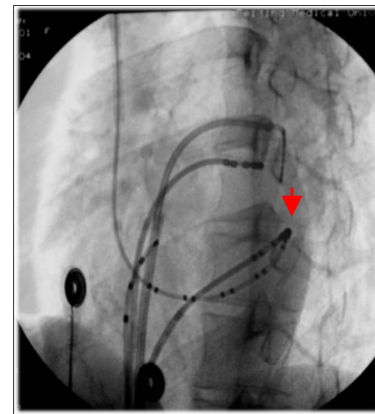

(a)

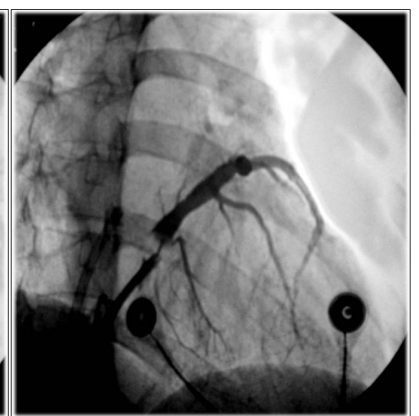

(b)
Figure 3. Left anterior oblique ((a) and (b)) chest X-rays showing the successful ablation site (arrows at the distal end of the ablation catheter). The site of the earliest atrial signal recording within the coronary sinus and where the first transient successful radiofrequency pulse was delivered is shown.

atrium, the majority originate from the pulmonary vein ostia, mitral annulus, left atrial appendage, and left septum being less common, even can originated from superior vena cava and in coronary sinus [4,6-17]. Patients with atrial tachycardia may experience a spectrum of symptoms like palpitations, dizziness, chest pain, dyspnea, fatigue, and syncope. The atrial rate during focal AT usually ranges between 130 and 250 beats/min, but may be as low as 100 beats/min or as high as 300 beats/min. In general, younger patients tend to have faster AT, with rates up to 340 beats/min in infants [18].

In our case, The ECG aspect of atrial tachycardia could associate negative $\mathrm{P}$ wave on leads I, II, III, aVF, and V5 to V6, positive on lead V1, and diphasic on lead aVL, the anticipated origin were left atrial, although the precise mapping was performed in left inferior pulmonary vein and mitral annulus, however, no target point was found ahead of CS1-2, the tried discharge was invalid. Under this condition, mapping and ablation inside the coronary sinus should be considered whenever the earliest endocardial atrial activation is recorded along the mitral annulus. However, the complication of ablation in coronary sinus including stenosis, perforation, thrombosis. In our patient, there have $20 \%$ stenois in coronary sinus. In order to prevent thrombosis, the anticoagulation drug aspirin been used for 3 months.

The management of focal AT has progressed rapidly in recent times. More recently the success of radiofrequency ablation has seen a shift toward a primary ablative approach in many patients. Focal AT is an uncommon arrhythmia and there remains a paucity of trials comparing the efficacy of different treatment regimes. In clinical practice, the different symptom may mislead the correct diagnosis, delayed the prompt therapy strategy. So we should pay attention to the possibility of atrial tachycardia, EP study if necessary. Only in this way, we can reduce the probability of misdiagnosis.

\section{REFERENCES}

[1] Roberts-Thomson, K.C., Kistler, P.M. and Kalman, J.M. (2006) Focal atrial tachycardia I: Clinical features, diagnosis, mechanisms, and anatomic location. Pacing and Clinical Electrophysiology, 29, 643-652. doi:10.1111/j.1540-8159.2006.00413.x

[2] Lesh, M.D., Kalman, J.M. and Olgin, J.E. (1996) New approaches to treatment of atrial flutter and tachycardia. Journal of Cardiovascular Electrophysiology, 7, 368381. doi:10.1111/j.1540-8167.1996.tb00539.x

[3] Chen, S.A., Tai, C.T., Chiang, C.E., Ding, Y.A. and Chang, M.S. (1998) Focal atrial tachycardia: Reanalysis of the clinical and electrophysiologic characteristics and prediction of successful radiofrequency ablation. Journal of Cardiovascular Electrophysiology, 9, 355-365. doi:10.1111/j.1540-8167.1998.tb00924.X

[4] Kistler, P.M., Roberts-Thomson, K.C., Haqqani, H.M., Fynn, S.P., Singarayar, S., Vohra, J.K., Morton, J.B., Sparks, P.B. and Kalman, J.M. (2006) P-wave morphology in focal atrial tachycardia: Development of an algorithm to predict the anatomic site of origin. Journal of the American College of Cardiology, 48, 1010-1017. doi:10.1016/j.jacc.2006.03.058

[5] Navarrete, A.J., Arora, R., Hubbard, J.E. and Miller, J.M. (2003) Magnetic electroanatomic mapping of an atrial tachycardia requiring ablation within the coronary sinus. Journal of Cardiovascular Electrophysiology, 14, 13611364. doi:10.1046/j.1540-8167.2003.03334.x

[6] Kistler, P.M., Sanders, P., Fynn, S.P., Stevenson, I.H., Hussin, A., Vohra, J.K., Sparks, P.B. and Kalman, J.M. (2003) Electrophysiological and electrocardiographic characteristics of focal atrial tachycardia originating from the pulmonary veins: Acute and long-term outcomes of radiofrequency ablation. Circulation, 108, 1968-1975. doi:10.1161/01.CIR.0000095269.36984.75

[7] Kistler, P.M., Sanders, P., Hussin, A., Morton, J.B., Vohra, J.K., Sparks, P.B. and Kalman, J.M. (2003) Focal atrial tachycardia arising from the mitral annulus: Electrocardiographic and electrophysiologic characterization. Journal of the American College of Cardiology, 41, 22122219. doi:10.1016/S0735-1097(03)00484-4

[8] Marrouche, N.F., SippensGroenewegen, A., Yang, Y., Dibs, S. and Scheinman, M.M. (2002) Clinical and electrophysiologic characteristics of left septal atrial tachycardia. Journal of the American College of Cardiology, 40, 1133-1139. doi:10.1016/S0735-1097(02)02071-5

[9] Gonzalez, M.D., Contreras, L.J., Jongbloed, M.R., Rivera, J., Donahue, T.P., Curtis, A.B., Bailey, M.S., Conti, J.B., Fishman, G.I., Schalij, M.J. and Gittenberger-de-Groot, A.C. (2004) Left atrial tachycardia originating from the mitral annulus-aorta junction. Circulation, 110, 3187-3192. doi:10.1161/01.CIR.0000147613.45259.D1

[10] Wang, Y.L., Li, X.B., Quan, X., Ma, J.X., Zhang, P., Xu, Y., Zhang, H.C. and Guo, J.H. (2007) Focal atrial tachycardia originating from the left atrial appendage: electrocardiographic and electrophysiologic characterization and long-term outcomes of radiofrequency ablation. 
Journal of Cardiovascular Electrophysiology, 18, 459464. doi:10.1111/j.1540-8167.2007.00808.x

[11] Roberts-Thomson, K.C., Kistler, P.M., Haqqani, H.M., McGavigan, A.D., Hillock, R.J., Stevenson, I.H., Morton, J.B., Vohra, J.K., Sparks, P.B. and Kalman, J.M. (2007) Focal atrial tachycardias arising from the right atrial appendage: Electrocardiographic and electrophysiologic characteristics and radiofrequency ablation. Journal of Cardiovascular Electrophysiology, 18, 367-372. doi:10.1111/j.1540-8167.2006.00754.x

[12] Kistler, P.M., Fynn, S.P., Haqqani, H., Stevenson, I.H., Vohra, J.K., Morton, J.B., Sparks, P.B. and Kalman, J.M. (2005) Focal atrial tachycardia from the ostium of the coronary sinus: Electrocardiographic and electrophysiological characterization and radiofrequency ablation. Journal of the American College of Cardiology, 45, 1488-1493. doi:10.1016/j.jacc.2005.01.042

[13] Badhwar, N. Kalman, J.M., Sparks, P.B., Kistler, P.M., Attari, M., Berger, M., Lee, R.J., Sra, J. and Scheinman, M.M. (2005) Atrial tachycardia arising from the coronary sinus musculature: Electrophysiological characteristics and long-term outcomes of radiofrequency ablation. Journal of the American College of Cardiology, 46, 19211930. doi:10.1016/j.jacc.2005.07.042

[14] Yamabe, H., Tanaka, Y., Okumura, K., Morikami, Y., Kimura, Y., Hokamura, Y. and Ogawa, H. (2005) Electrophysiologic characteristics of verapamil-sensitive atrial tachycardia originating from the atrioventricular an- nulus. American Journal of Cardiology, 95, 1425-1430. doi:10.1016/j.amjcard.2005.02.007

[15] Yamada, T., Murakami, Y., Muto, M., Okada, T., Okamoto, M., Toyama, J., Yoshida, Y., Tsuboi, N., Ito, T., Kondo, T., Inden, Y., Hirai, M. and Murohara, T. (2004) Electrophysiologic characteristics of atrial tachycardia originating from the right pulmonary veins or posterior right atrium: double potentials obtained from the posterior wall of the right atrium can be useful to predict foci of atrial tachycardia in right pulmonary veins or posterior right atrium. Journal of Cardiovascular Electrophysiology, 15, 745-751. doi:10.1046/j.1540-8167.2004.03513.x

[16] Frey, B., Kreiner, G., Gwechenberger, M. and Gossinger, H.D. (2001) Ablation of atrial tachycardia originating from the vicinity of the atrioventricular node: significance of mapping both sides of the interatrial septum. Journal of the American College of Cardiology, 38, 394400. doi:10.1016/S0735-1097(01)01391-2

[17] Zhao, Z., Li, X. and Guo, J. (2009) Electrophysiologic characteristics of atrial tachycardia originating from the superior vena cava. Journal of Interventional Cardiac Electrophysiology, 24, 89-94. doi:10.1007/s10840-008-9324-0

[18] Mehta, A.V. and Ewing, L.L. (1993) Atrial tachycardia in infants and children: electrocardiographic classification and its significance. Pediatric Cardiology, 14, 199-203. doi:10.1007/BF00795370 